\title{
Vivaldi Antenna for UWB Applications: A Review
}

\author{
Mayank Sharma \\ Research Scholar, EEE, School of Engineering \& Technology, \\ Poornima University, Jaipur, India \\ msecajmer@gmail.com \\ Dr. Sunil Kumar Gupta \\ Professor, EEE, School of Engineering \& Technology, Poornima University, Jaipur, India \\ sunil.gupta@poornima.edu.in
}

\author{
Article Info \\ Page Number: $69-71$ \\ Publication Issue: \\ Vol 71 No. 1 (2022)
}

Article History

Article Received: 18 November 2021

Revised: 01 December 2021

Accepted: 15 December 2021

Publication: 27 January 2022

\begin{abstract}
This particular document explains the look associated with Vivaldi antenna as well as display various giving methods that's accustomed to provide give food to towards the tapered position Vivaldi Antenna. With this record, the actual antenna guidelines as well as materials qualities tend to be talked about to have UWB needs. This short article provides the actual books evaluation upon UWB technology utilizing various feed method.
\end{abstract}

Keywords: - Vivaldi Antenna, Antipodal Vivaldi Antenna (AVA).

\section{INTRODUCTION}

Since the fast development within the cellular conversation techniques, it takes the actual systems along with add-on functions and then the Ultra-Wide Band (UWB) technologies happen to be thoroughly used to supply extra functions [1-3]. Execute, cell phone corporations including apple company, Samsung, for example. Usually are creations UWB permitted motherboards with mobile phone devices which are widely-used the pros and additional highlights of UWB technological know-how [4-5]. Since the need with regard to transmit as well as cellular conversation systems is actually growing daily, the need associated with planning for a brand new kind of antenna along with functions such as wider impedance bandwidth as well as extremely directive the radiation design offers elevated. Directional antennas tend to be mainly utilized in programs to improve the actual cellular systems' capability and also to slow up the impact associated with co-channel disturbance as well as multipath results. Wideband antennas are utilized in various programs such as satellite television conversation, RADAR, microwave image resolution techniques, remote control realizing techniques, GPR recognition, as well as healthcare programs. However, wideband antennas will also be discovered helpful within broadband conversation to change the requirement with regard to several antennas with regard to varied programs because of its functions such as much less intricacy, reduce energy usage, along with a scaled-down impact. Vivaldi antennas are actually observed while is just about the suited prospects for the broadband directional transmission technique [5-8].

Year 1979 witnessed the creation of Vivaldi antenna by Gibson, which unfortunately belongs to the elegance for end-fire going antennas. Any Vivaldi antenna provides the offer associated with a slit path wherein benefit split within the slit path lengthens on top of $\mathrm{X} / 2$, the place by is certainly along any parameter [6]. Because of the changeover within the giving framework, the actual antenna creates reduced Sss reduction more than a comprehensive rate of recurrence variety. The actual antenna additionally offers controlled column thickness because of tapering from the position collection within the rapid type. To lessen alongside it lobe obtain, the finish from the antenna ought to be bent. You will find 3 places specifically give food to region, transit region, as well as the radiation region present in Vivaldi antenna. With regard to electromagnetic coupling, the actual give input to region is actually accountable. Some sort of improved design on the antenna was made by means of Gazit to help triumph over this con on the ETSA, which is often known as this AVA [7]. 
https://doi.org/10.17762/msea.v71i1.41

\section{FEED MECHANISM}

The actual Bandwidth is actually unlimited within Vivaldi antenna. The actual Bodily dimension from the antenna and also the manufacturing abilities would be the restriction about the bandwidth. The correct giving framework is important to maximize the actual bandwidth. Various inputs are utilized. Both input methods tend to be straight combined changeover as well as electromagnetically combined changeover. The techniques that are popular would be the coaxial collection give food to and also the microstrip collection give food to. The actual Microstrip in order to position collection changeover may be the moderate greatest for that giving the actual structure. The actual Position collection is actually about the 1 aspect from the substrate and also the microstrip is actually etched upon an additional aspect from the substrate. The actual Microstrip as well as slot-lines traversing one another from correct position within their structure. Once the slot-lines as well as strip-line traversing one another, the actual slot machines tend to be stretches through $\lambda \mathrm{s} / 4$ past the actual microstrip as well as $\lambda \mathrm{m} / 4$ past the actual position collection. The primary disadvantage is actually decreased in the actual bandwidth however enhanced rays design [6-7].

Within Vivaldi antennas, the ability move in between the input as well as antenna is performed via a given feed and its proper matching. Generally, the actual impedance of the matched circuit is actually $50 \Omega$. Through optimum energy move theorem, the actual Vivaldi antenna ought to be given in a stage exactly where enter impedance is actually $50 \Omega$ with regard to complete enter energy. There are numerous giving processes for the actual Vivaldi antenna to complement this problem. A tapered microstrip line feed with width of port to be $1.5 \mathrm{~mm}$ is utilized in order to maintain or have a good matching of impedance. The transition of feed in the in the AVA structure leads to bandwidth enhancement along with reduction in the insertion loss [8-9].

\section{ANTIPODAL VIVALDI ANTENNA: CONCEPT OF BALANCING AND UNBALANCING}

The actual BAVA because suggested through Langley et ing. [10] includes a good ultra-wide bandwidth changeover as well as overcomes poor polarization overall performance from the fundamental out of balance antipodal type. The actual framework associated with BAVA includes 3 copper levels using the external 2 copper levels behaving because bottom levels and also the center coating like a conductor. Just about all copper levels tend to be divided through substrates. The actual framework equipoise the actual launching associated with dielectric materials between conductor as well as floor airplane. For this reason managing, modifications within column path because of rate of recurrence, polarization, or even alignment. The lengthy substrate with slots resulted in improved directive performance and enhanced the $\mathrm{F}$ to $\mathrm{B}$ ratio [11].

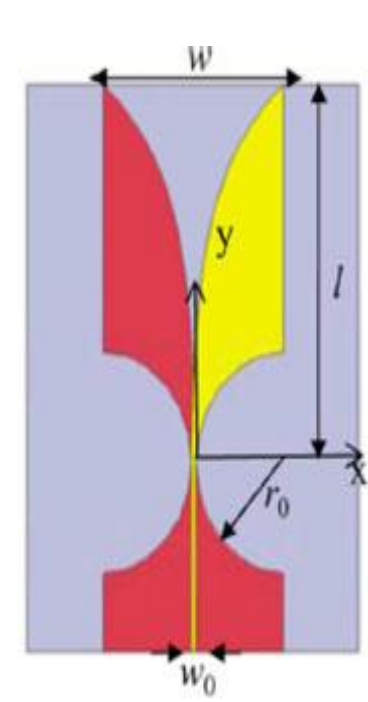

(a)

\section{VIVALDI ANTENNA For BANDWIDTH ENHANCEMENT}

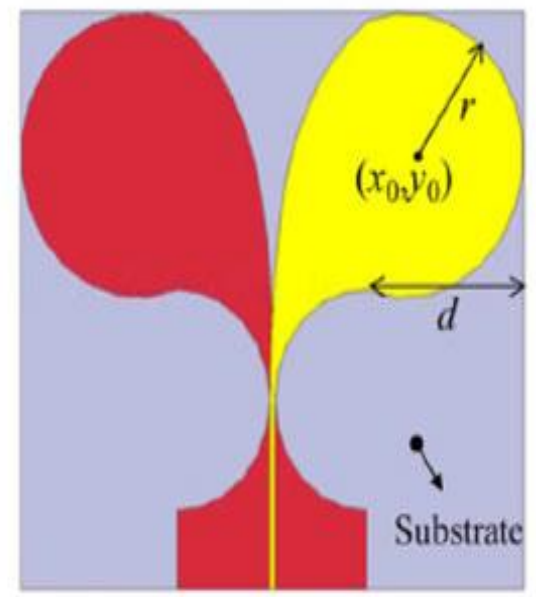

(b)

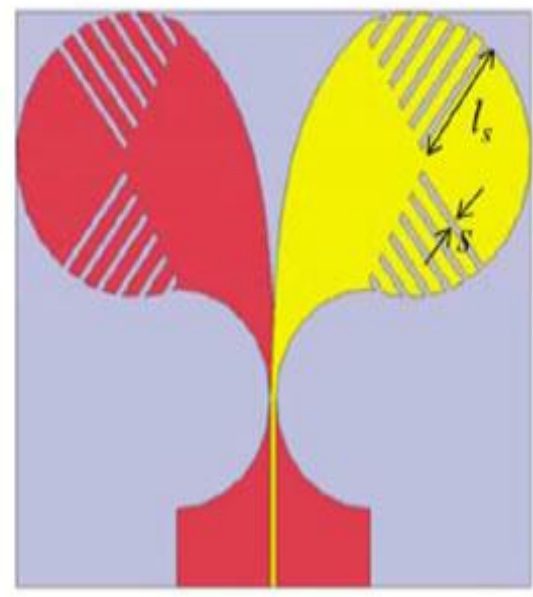

(c)

Fig. 1: Targeting UWB applications with Vivaldi antenna [12]

It concept was utilized in distinctive captivating functions, which includes biomedical detection, ground-penetrating radar, etc. UWB contact products and even UWB houseplants position products contain gained vital creation thanks to delightful components on high-speed transmitting speed and even increased multipath option file size. At the moment Vivaldi antenna accomplished major treatment thanks to various captivating includes which includes planar building; very easy manufacturing that is definitely traditionally designed for UWB functions.

Due to the inexpensive as well as easy integration, Vivaldi antenna is among the greatest applicants utilized in the majority of the 
modern-day UWB techniques. Nevertheless, a few of the antennas are afflicted by a sizable framework, higher cross-polarization, giving changeover restrictions in addition to higher side-lobes. The actual antenna proven within Fig. 1 can be used within UWB. With this style [12], 2 circular-shaped notches tend to be additional upon 2 sides from the antenna, because proven within Fig. 1(b). Slot machines associated with various measures tend to be etching upon each hand from the antenna to enhance the radiation design as well as directivity from the antenna, because proven within Fig. 1(c). The equation associated with the tapering of slot is given below as:

$$
x=+\left[w_{0}-\frac{1}{2} w_{0} e^{(\not \gamma Y)}\right]
$$

.For that best as well as base levels, exactly where w0 may be the thickness from the microstrip collection as well as $\alpha$ may be the rapid changeover price. The actual cut-off wavelength from the antenna depends upon the actual aperture thickness from the antenna which may be indicated as:

$$
\lambda_{e}=2 w
$$

Exactly where w may be the aperture thickness from the antenna as well as $\lambda c$ may be the cut-off wavelength. The actual round form of flare along with slot improves bandwidth as well as low-frequency overall performance from the antenna.

\section{CONCLUSION}

Vivaldi antenna is among the majority of guaranteeing applicants with regard to UWB programs because of its broad bandwidth as well as showcased directive the radiation features. A comprehensive overview of just about all Vivaldi antenna overall performance enhancement techniques contained in the majority of the literatures may be reported with this document. These types of techniques provide the actual customization in line with the bodily geometry of the antenna that leads to a big change in a variety of antenna guidelines such as dimension, obtain, entrance in order to back again percentage, bandwidth, the radiation design, polarization, as well as working rate of recurrence variety.

\section{REFERENCES}

[1] U.S. - F. C. C. "F. C. C. - N. O. I.: Rules Regarding ultra-wide-band transmission systems", E T Docket No. 98 - 153 , Sept. 1998.

[2] Antenna theory: analysis and design, Constantine A. Balanis, third edition, Hoboken, NJ: Wiley, 2005, ISBN 047166782X (hbk.).

[3] M. Ojaroudi, C. Ghobadi, and J. Nourinia "A Small Square Monopole Antenna with an Inverted TShaped Notch in the Ground Plane for U W B Application" IEEE Antennas and Wireless Propagation Letters, V O L. 8, pp. 728-731, 2009.

[4] Mayank Sharma and Dr. Sunil Kumar Gupta, "Design of a Planar Ultra Wide Band Antenna Structure using Stepped Contours" Turkish Journal of Computer and Mathematics Education, ISSN: 1309-4653, Vol.12 No.6 (2021), Vol-12, Issue No. 6, Page No. 3875-3881, April 2021.

[5] H. Mewara, M. Sharma, M. Sharma, M. Gupta, A. Dadhich, "Planar ultrawideband antenna design using circular shaped truncated corners and notches," Proceedings of 2nd International Conference Computer and Communication Technologies (IC3T 2015) at CMR Technical Campus, Hyderabad on 24 - 26 July, 2015. DOI: 10.1007/978-81-322-2517-1_68.

[6] Gibson PJ (1979) The Vivaldi aerial. Proceedings of the 9th European Microwave Conference, pp. 101-105.

[7] Gazit E (1988) Improved design of the Vivaldi antenna. IEEE Proceedings 135, 89-92.

[8] Liu Y, Zhou W, Yang S, Li W, Li P and Yang S (2016) A novel miniaturized Vivaldi antenna using tapered slot edge with resonant cavity structure for ultrawideband applications. IEEE Antennas and Wireless Propagation Letters 15, 1881-1884.

[9] Wang Z, Yin Y, Wu J and Lian R (2016) A miniaturized CPW-fed antipodal Vivaldi antenna with enhanced radiation performance for wideband applications. IEEE Antennas and Wireless Propagation Letters 15, 16-19.

[10] Langley J, Hall P and Newham P (1993) Novel ultra wide-bandwidth Vivaldi antenna with low cross polarisation. Electronics Letters 29, 2004-2005.

[11] Wang P, Zhang H, Wen G and Sun Y (2012) Design of modified balanced antipodal Vivaldi antenna. Progress in Electromagnetics Research C 25, 271-285

[12] Bai J, Shi S and Prather DW (2011) Modified compact antipodal Vivaldi antenna for 4-50-GHz UWB application. IEEE Transactions on Microwave Theory and Techniques 59, 1501-1507. 